\title{
Time Delay in Thin Dielectric Slabs with Saturable Nonlinearity
}

\author{
P.P. BeliČev ${ }^{a, *}$, I. Ilić ${ }^{a}$, J. Radovanović $^{b}$, V. Milanović ${ }^{b}$ And LJ. HadŽIevshi ${ }^{a}$ \\ ${ }^{a}$ Vinca Institute of Nuclear Sciences, Atomic Physics Laboratory \\ Mike Petrovića Alasa 12-14, P.O. Box 522, 11001 Belgrade, Serbia \\ ${ }^{b}$ Faculty of Electrical Engineering \\ Bulevar kralja Aleksandra 73, 11120 Belgrade, Serbia
}

\begin{abstract}
Time delays for an intense transverse electric wave propagating through a slab with saturable nonlinearity are investigated. The nonlinearity is assumed in a form of the Vinetskii-Kukhtarev model, which is relevant for the slabs made of nonlinear photorefractive crystals, such as $\mathrm{GaAs}$ and $\mathrm{LiNbO}_{3}$, which feature a saturable nonlinearity. The expressions for the group delay and the dwell time are derived and the relation between them is studied. It is shown that the difference between them has three different contributions. The first one corresponds to the self-interference associated with the dispersion of the medium surrounding the slab. The other two appear due to the nonlinearity of the slab and oblique incidence of the transverse electric wave. All the results are compared with the case of dielectric slabs with cubic (Kerr) nonlinearity.
\end{abstract}

PACS numbers: 03.65.Xp, 41.20.Jb, 42.25.Bs, 42.70.Nq

\section{Introduction}

The question of "how long it takes for a wave packet to tunnel through a potential barrier" has been occupying physicists for a long time. The deep relationship between the Schrödinger and the Helmholtz equation, as well as the analogy between the quantum-mechanical tunneling of particles and the classical electromagnetic tunneling of evanescent waves $[1,2]$, enable determination of tunneling times. Condon was the first scientist who pointed out to the problem of the speed of tunneling in 1931 [3]. Years later, Hartman showed that time delay saturates with increasing barrier lengths [4].

Numerous experiments [5-7] have revealed the presence of superluminal velocities for wave packets tunneling through waveguide with positive index of refraction. In materials with regions of anomalous dispersion, these velocities can even be negative. This phenomenon can be explained by the fact that the peak of the transmitted wave packet would emerge prior to the peak of the incident wave packet entering the medium. These negative velocities do not, as it may seem, violate causality, because energy propagation velocity inside the medium is positive and smaller than the speed of light in vacuum c. For this reason, it is necessary to establish a new, appropriate time scale with which one characterizes the tunneling of electromagnetic waves. Two tunneling time

* corresponding author; e-mail: petrab@vin.bg.ac.yu definitions - the group delay and the dwell time - are considered to be the most appropriate [8]. The relation between these two time definitions has been suggested by Winful [9]: the group delay is a sum of the dwell time and a term called self-interference time, which represents the time that a wave packet spends interfering with the reflected wave in front of an obstacle (this time is non-zero only when background medium is dispersive). However, this relation was valid only for an obstacle made of linear, non-absorptive, non-magnetic material with normal incidence. Later on, tunneling times have been calculated for all linear [10] and media with cubic nonlinearity [11].

Nowadays, materials with saturable nonlinearity attract great attention because of their characteristic to lead numerous physic quantities to saturation [12-14]. The goal of this paper is to derive an explicit expression for the mentioned tunneling times, i.e. group delay and the dwell time, when the wave packet is tunneling through an obstacle made of material with saturable nonlinearity. All derivations in this paper presume propagation of transverse electric (TE) modes. Numerical calculations were carried out for different nonlinear materials and compared to those obtained for the slabs with the Kerr type of nonlinearity, presented in [11].

\section{Theoretical considerations}

The model of a planar dielectric waveguide and an obstacle made of a nonlinear material placed inside the waveguide is shown in Fig. 1. Both the waveguide (whose 
permittivity is $\varepsilon_{b}$ ) and the obstacle are made of non-magnetic materials. The thickness of the obstacle is denoted by $L$. Since the obstacle is made of a material with saturable nonlinearity, its permittivity $\varepsilon_{\mathrm{NL}}$ is given by:

$$
\varepsilon_{\mathrm{NL}}=\varepsilon_{\mathrm{LN}}+\alpha \frac{\left|E_{y}\right|^{2} / E_{\mathrm{c}}^{2}}{1+\kappa\left|E_{y}\right|^{2} / E_{\mathrm{c}}^{2}}=\varepsilon_{\mathrm{LN}}+\varepsilon_{\mathrm{SLN}},
$$

where $\varepsilon_{\mathrm{LN}}$ represents the linear part of the permittivity and $\varepsilon_{\mathrm{SNL}}$ is a saturable contribution to the permittivity.

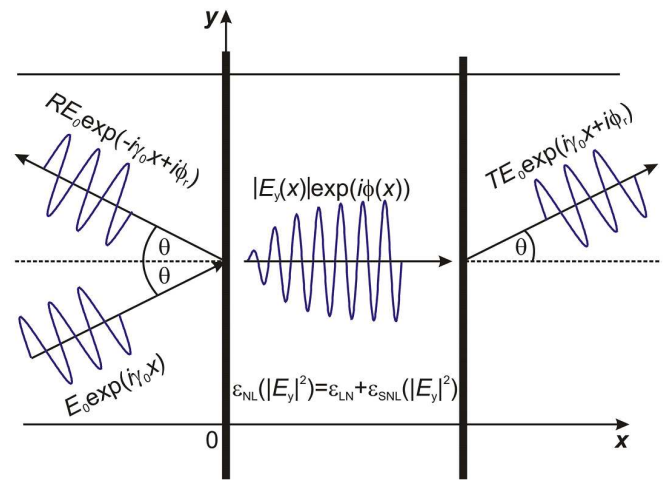

Fig. 1. The model.

Here, $\alpha= \pm 1$, depending on focusing or defocusing nonlinearity, $E_{y}$ is the $y$ component of the electric field inside the obstacle, $E_{\mathrm{c}}$ is a characteristic electric field and $\kappa$ stands for the saturation strength. In this paper, we presume an obstacle placed in vacuum.

Assuming that the waveguide is finite only in $y$ direction and that the wave packet propagates along the $x$ axis, with an angle of incidence $\theta$, the Helmholtz nonlinear equation within the obstacle reads

$$
E_{y}^{\prime \prime}+\left(k_{0}^{2} \varepsilon_{\mathrm{NL}}-\beta^{2}\right) E_{y}=0,
$$

where $k_{0}$ is the vector of propagation and $\beta=$ $\sqrt{\varepsilon_{b} \mu_{b}} k_{0} \sin (\theta)$ stands for the propagation constant. By switching to the dimensionless form, via replacing $\rho^{2}=$ $k_{0}^{2} \varepsilon_{\mathrm{LN}}-\beta^{2}, \Omega=2 \kappa \rho^{2} /\left(k_{0}^{2} \alpha\right), E_{y}=\rho / k_{0} \sqrt{2 E_{\mathrm{c}}^{2} / \alpha} y$ and $\xi=\rho x$, the Helmholtz equation can be rewritten as

$$
y_{\xi}^{\prime \prime}+\left(1+2 \frac{|y|^{2}}{1+\Omega|y|^{2}}\right) y=0 .
$$

Taking the solution as $y(\xi)=r(\xi) \mathrm{e}^{\mathrm{i} \phi(\xi)}$ and integrating the above expression, we obtain

$$
\frac{\left(S^{\prime}\right)^{2}}{4 S}+\frac{\Theta^{2}}{S}+S+\frac{2 S}{\Omega}-\frac{2 \ln (1+\Omega S)}{\Omega^{2}}+C_{1}=0,
$$

where $S=r^{2}, \Theta=\gamma_{0}\left|E_{0}\right|^{2} k_{0}^{2} \alpha\left(1-|R|^{2}\right) /\left(2 E_{\mathrm{c}}^{2} \rho^{3}\right), \gamma_{0}=$ $k_{0} \sqrt{\varepsilon_{b}} \cos (\theta)$ and $C_{1}$ is the integration constant. After inserting the boundary conditions on the cross-sections waveguide-obstacle and obstacle-waveguide into the previous expression, we derive the following three equations:

$$
C_{1}=-\frac{\left(S^{\prime}(0)\right)^{2}}{4 S(0)}-\frac{\Theta^{2}}{S(0)}-S(0)-\frac{2 S(0)}{\Omega}
$$

$$
\begin{aligned}
& +\frac{2 \ln (1+\Omega S(0))}{\Omega^{2}}, \\
& C_{1}=-\frac{\Theta^{2}}{S(L)}-S(L)-\frac{2 S(L)}{\Omega} \\
& \quad+\frac{2 \ln (1+\Omega S(L))}{\Omega^{2}}, \\
& \int_{\mathrm{S}(0)}^{S(\xi L)} \frac{\mathrm{d} t}{\sqrt{-4 t^{2}-4 t C_{1}-4 \Theta^{2}-\frac{8 t^{2}}{\Omega}+\frac{8 t}{\Omega^{2}} \ln (1+\Omega t)}} \\
& =\xi L,
\end{aligned}
$$

where $S(0)$ and $S(L)$ represent the squares of the electric field amplitude on the obstacle boundaries, and are functions of the amplitude and the argument $\phi_{\mathrm{r}}$ of the complex reflection coefficient (i.e. $|R|)$. Solving of this system of three equations provides $C_{1},|R|$, and $\phi_{\mathrm{r}}$, which further enables determining of $S(\xi)$ from differential Eq. (4). Obviously, this implies defining of $E_{y}$ and subsequent expressions for the dwell time and the group delay, which is the goal of this paper.

Dwell time is a measure of the time spent by a wave packet in a given region of space, and is defined as $[9,15,16]$ :

$$
\tau_{\mathrm{d}}=\frac{\langle W\rangle}{\left\langle P_{\text {in }}\right\rangle},
$$

where $W$ stands for the stored electromagnetic energy inside the barrier and $P_{\text {in }}$ represents the time averaged incident power, which is equal to $\left\langle P_{\text {in }}\right\rangle=$ $\left[\sqrt{\varepsilon_{b}} \varepsilon_{0} c \cos (\theta) A / 2\right]\left|E_{0}\right|^{2}$. Here, $\varepsilon_{0}$ is the permittivity of vacuum, $A$ stands for the cross-section surface perpendicular to the $x$ axis, and $c$ is the speed of light in vacuum.

For a media with saturable nonlinearity, the electromagnetic energy represented by the sum of the electric energy $W_{\mathrm{e}}$ and the magnetic energy $W_{\mathrm{m}}$, can be derived as

$$
\begin{gathered}
\langle W\rangle=\left\langle W_{\mathrm{e}}\right\rangle+\left\langle W_{\mathrm{m}}\right\rangle=\frac{A \varepsilon_{0}}{2} \int_{0}^{L} \varepsilon_{\mathrm{NL}}\left|E_{y}\right|^{2} \mathrm{~d} x \\
-\frac{A \gamma_{0}\left|E_{0}\right|^{2}}{2 \omega^{2} \mu_{0}} \operatorname{Im}(R)+\frac{A \varepsilon_{0}}{2} \frac{\omega}{2} \int_{0}^{L} \frac{\mathrm{d} \varepsilon_{\mathrm{LN}}}{\mathrm{d} \omega}\left|E_{y}\right|^{2} \mathrm{~d} x .
\end{gathered}
$$

Here, $\omega$ is the incident wave frequency and $\mu_{0}$ represents the permeability of vacuum.

By inserting the expressions for $\langle W\rangle$ and $\left\langle P_{\text {in }}\right\rangle$ into (8), followed by some basic simplifications, we arrive to

$$
\begin{aligned}
& \tau_{\mathrm{d}}=\frac{\int_{0}^{L}\left(\frac{\omega}{2} \frac{\mathrm{d} \varepsilon_{\mathrm{LN}}}{\mathrm{d} \omega}+\varepsilon_{\mathrm{LN}}\right)\left|\frac{E_{y}}{E_{0}}\right|^{2} \mathrm{~d} x}{\sqrt{\varepsilon_{b}} c \cos (\theta)} \\
&+\alpha \frac{\int_{0}^{L} \frac{\left|E_{y}\right|^{2}}{E_{\mathrm{c}}^{2}+\left|E_{y}\right|^{2}}\left|\frac{E_{y}}{E_{0}}\right|^{2} \mathrm{~d} x}{\sqrt{\varepsilon_{b}} c \cos (\theta)}-\frac{\operatorname{Im}(R)}{\omega} .
\end{aligned}
$$

This expression is valid for dispersive nonlinear media. The first term in this expression represents the influence of the linear part of the obstacle's permittivity, while the second term represents the influence of the saturable nonlinearity in the obstacle. 
Subtracting the conjugate of the Helmholtz equation multiplied by $\mathrm{d} E_{y} / \mathrm{d} \omega$ from the derivative of the Helmholtz equation (with respect to $\omega$ ) multiplied by $E_{y}^{*}$, and integrating the obtained expression along the barrier, results in a connection between the group delay and the dwell time

$$
\begin{gathered}
\tau_{\mathrm{g}}=\varepsilon_{\theta} \tau_{\mathrm{d}}+\left(\frac{1}{\omega} \varepsilon_{\theta}-\frac{1}{\gamma_{0}} \frac{\mathrm{d} \gamma_{0}}{\mathrm{~d} \omega}\right) \operatorname{Im}(R) \\
+\frac{\int_{0}^{L} \varepsilon_{\mathrm{SNL}}\left|\frac{E_{y}}{E_{0}}\right|^{2} \mathrm{~d} x}{\sqrt{\varepsilon_{b}} c \cos (\theta)} \\
-\frac{\varepsilon_{\mathrm{LN}}^{+}}{\varepsilon_{\mathrm{LN}}^{+}-\sin ^{2}(\theta) \varepsilon_{b}^{+}} \frac{\int_{0}^{L} \varepsilon_{\mathrm{SNL}}^{+}\left|\frac{E_{y}}{E_{0}}\right|^{2} \mathrm{~d} x}{\sqrt{\varepsilon_{b}} c \cos (\theta)},
\end{gathered}
$$

where we have denoted $\varepsilon_{\theta}=1-\sin ^{2}(\theta) \varepsilon_{b}^{+} / \varepsilon_{\mathrm{LN}}^{+}$, and $\varepsilon^{ \pm}=\varepsilon \pm \omega / 2 \cdot \mathrm{d} \varepsilon / \mathrm{d} \omega=\varepsilon[1 \pm \omega /(2 \varepsilon) \cdot \mathrm{d} \varepsilon / \mathrm{d} \omega]$. The third term in previous expression represents the nonlinear time $\tau_{\mathrm{NL}}$, which is the explicit contribution of the nonlinearity. The fourth term, marked as $\tau_{\mathrm{t}}$, appears due to the oblique incidence of the TE wave.

In case of linear, non-dispersive obstacle, placed inside the dispersive waveguide, i.e. $\mathrm{d} \varepsilon_{\mathrm{LN}} / \mathrm{d} \omega=0, \mathrm{~d} \varepsilon_{b} / \mathrm{d} \omega \neq 0$, and with $\theta=0^{\circ}, \tau_{\mathrm{NL}}$ and $\tau_{\mathrm{t}}$ vanish, so the expression for the group delay reduces to the expression that Winful derived in [9]. He presented the group delay as a sum of the dwell time and a term known as the self-interference time, which equals $\tau_{\mathrm{i}}=[\operatorname{Im}(R) / \beta](\beta / \omega-\mathrm{d} \beta / \mathrm{d} \omega)$. Hence, this result implies the validity of the expression (11) derived in this paper.

\section{Numerical results and discussion}

In this paper, the numerical results show dependences of $\tau_{\mathrm{d}}, \tau_{\mathrm{g}}, E_{y}$ and $|T|$ on different values of the amplitude of incident electric field $E_{0}$, with angle of incidence and incident wavelength chosen to be $\theta=10^{\circ}$ and $\lambda=10^{-6} \mathrm{~m}$, respectively. The comparison among these results and those presented in [11] is given. The parameters of the obstacle's permittivity $E_{\mathrm{c}}$ and $\kappa$, as well as its length $L$, are chosen in order to attain best effect results possible, and are equal to $E_{\mathrm{c}}=1 \mathrm{~V} / \mathrm{m}, \kappa=1$ and $L=10^{-7} \mathrm{~m}$.

The first case observes an obstacle made of self-focusing nonlinear material, i.e. $\alpha=1$. Figure 2 depicts the dependence between normalized electric field magnitude $\left|E_{y}\right| /\left|E_{\mathrm{t}}\right|$ and the length of the obstacle $x$, for three different values of $E_{0}$, i.e. when $E_{0}=0.05 \mathrm{~V} / \mathrm{m}$, $E_{0}=1 \mathrm{~V} / \mathrm{m}$ and $E_{0}=5 \mathrm{~V} / \mathrm{m}$. It is quite apparent that the amplitude of the electric field saturates with the length of the obstacle.

We should note that in case of an obstacle made of a self-defocusing material, i.e. $\alpha=-1$, and with comparable values of the incident electric field, similar shapes to those of Fig. 2 are obtained. However, in this type of media the values of $\left|E_{y}\right| /\left|E_{\mathrm{t}}\right|$ increase with the increase in $E_{0}$, contrary to the self-focusing media.

Comparative results for the group delay and the dwell time for a certain range of $E_{0}$, for self-focusing and self-

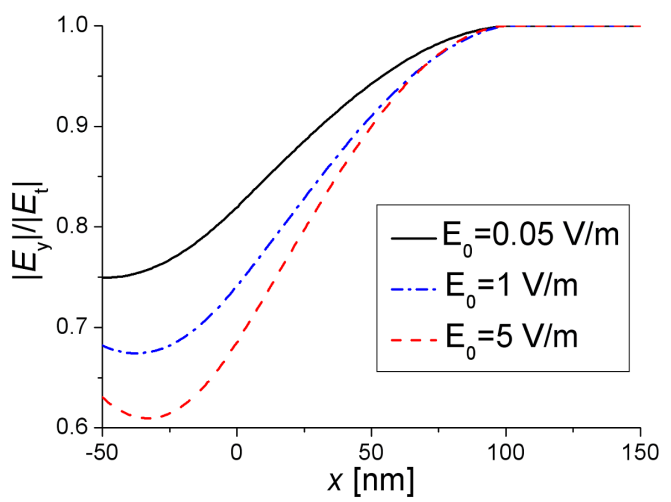

Fig. 2. Distribution of the normalized electric field amplitude $\left|E_{y}\right| /\left|E_{\mathrm{t}}\right|$ for three different values of the incident field amplitude $E_{0}$ and for $\alpha=+1$.

-defocusing medium, are given in Fig. 3. As it can be seen, for the same type of medium, these two times have different, but very close values. The distinction between them occurs because of the presence of $\tau_{\mathrm{NL}}$ and $\tau_{\mathrm{t}}$, whose relative influence is small (the difference is $\sim 10^{-18} \mathrm{~s}$ ), but nevertheless existing. In case of the normal incidence $\theta=0^{\circ}, \tau_{\mathrm{NL}}$ and $\tau_{\mathrm{t}}$ cancel each other out, so $\tau_{\mathrm{d}}$ becomes equal to $\tau_{\mathrm{g}}$. Also, the dwell time and the group delay have lower values for the self-focusing medium than for the self-defocusing one. The comparison between the results obtained for media with cubic (Kerr) nonlinearity [11], shows that for small incident field amplitudes, the values of tunneling times match those obtained in this paper. This arises from the fact that for small $E_{0}$, the expression which describes the permittivity for saturable nonlinearity reduces to the expression for cubic nonlinearity.

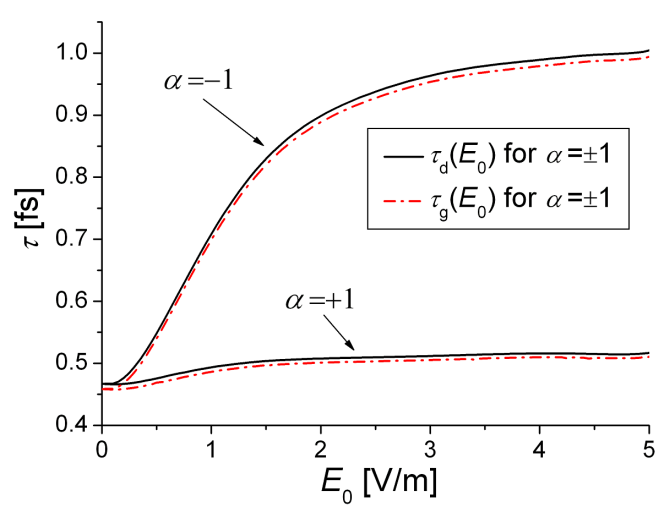

Fig. 3. Dwell time and group delay dependence on the incident field amplitude $E_{0}$, for $\alpha= \pm 1$.

In addition, the transmittivity as a function of $E_{0}$ was calculated for self-defocusing and self-focusing material. In case of a self-defocusing medium, $|T|$ saturates nearly to 1 , so the obstacle practically becomes totally transparent for electromagnetic (EM) waves. For self-focusing 
medium, however, transmittivity decreases with increasing $E_{0}$, and saturates at around 0.88. These results, especially the ones for self-defocusing media, are, also, comparable with those obtained for the Kerr nonlinearity media [11]. Again, for small values of $E_{0}$, the values of transmittivity coincide, however, for higher values of incident field amplitudes, it seems as if the transmittivity curve for self-defocusing media in our case follows the shape of the upper peaks of transmittivity curve in the cubic nonlinear media. This conclusion can be applied to the results for dwell time and the group delay, with the difference that, here, our results follow the shape of lower peaks in cubic nonlinearity case.

\section{Conclusion}

In this paper a more general expression that describes the relation between the dwell time and the group delay, and which is valid for nonlinear materials, is derived. An obstacle made of material with saturable nonlinearity and surrounded by air, was used as a model. It is shown that, for non-magnetic obstacle surrounded by air, these two times saturate and can be equal, if the angle of incidence is $0^{\circ}$. For other values of incidence angles there is an expression which, apart from these two quantities, includes two more times, namely - the self-interference time and the nonlinear time, though very small compared to the dwell time and group delay. Numerical results also show saturation of the electric field in the obstacle, as well as of the transmittivity. In contrast to the self-defocusing medium, the self-focusing medium gives minor values for all relevant quantities. The results show that the transmittivity saturates to lower value than its initial one for self-focusing media, while in self-defocusing it achieves its maximum value, i.e. 1. All the results are in good agreement with those obtained for the Kerr type media.

\section{Acknowledgments}

This work was supported by the Ministry of Science (Republic of Serbia). V.M. and J.R. would also like to acknowledge the financial support provided by the NATO Collaborative Linkage Grant (reference CBP.EAP.CLG 983316).

\section{References}

[1] Th. Martin, R. Landauer, Phys. Rev. A 45, 2611 (1992).

[2] R.Y. Chiao, P.G. Kwait, A.M. Steinberg, Physica B 175, 257 (1991).

[3] E.H. Condon, Rev. Mod. Phys. 3, 43 (1931).

[4] T.E. Hartman, J. Appl. Phys. 33, 3427 (1962).

[5] A. Enders, G. Nimtz, Phys. Rev. E 48, 632 (1993).

[6] A.M. Steinberg, P.G. Kwiat, R.Y. Chiao, Phys. Rev. Lett. 71, 708 (1993).

[7] Ch. Spielmann, R. Szipösz, A. Stingl, F. Krausz, Phys. Rev. Lett. 73, 2308 (1994).

[8] H.G. Winful, Phys. Rep. 436, 1 (2006).

[9] H.G. Winful, Phys. Rev. E 68, 016615 (2003).

[10] I. Ilić, P.P. Beličev, V. Milanović, J. Radovanović, J. Opt. Soc. Am. B 25, 1800 (2008).

[11] G. Isić, V. Milanović, J. Radovanović, Z. Ikonić, D. Indjin, P. Harrison, Phys. Rev. A 77, 033821 (2008).

[12] A. Maluckov, Lj. Hadžievski, N. Lazarides, G.P. Tsironis, Phys. Rev. E 77, 046607 (2008).

[13] Y.V. Kartashov, A.A. Egorov, V.A. Vysloukh, L. Torner, J. Opt. B 6, 279 (2004).

[14] Lj. Hadžievski, A. Maluckov, M. Stepić, D. Kip, Phys. Rev. Lett. 93, 033901 (2004).

[15] F.T. Smith, Phys. Rev. 118, 349 (1960).

[16] H.G. Winful, Phys. Rev. B 72, 046608 (2005). 